\title{
Surgical management of angiomyolipoma with vena cava thrombus during pregnancy: a case report
}

\author{
Zhenan Zhang ${ }^{1 \#}$, Zhe Liu ${ }^{2 \#}$, Lin Yao ${ }^{1}$, Qian Zhang ${ }^{1}$, Zhisong $\mathrm{He}^{1}$ \\ ${ }^{1}$ Department of Urology, Peking University First Hospital, Institute of Urology, Peking University, National Research Center for Genitourinary \\ Oncology, Beijing 100034, China; ${ }^{2}$ Department of Obstetrics and Gynecology, Peking University First Hospital, Beijing 100034, China \\ "These authors contributed equally to this work. \\ Correspondence to: Zhisong He. Department of Urology, Peking University First Hospital, Institute of Urology, Peking University, National Research \\ Center for Genitourinary Oncology, 8 Xishiku Street, Xicheng District, Beijing 100034, China. Email: hezhisongbjmu@126.com.
}

\begin{abstract}
Renal angiomyolipoma (AML) can grow and worsen during pregnancy. Sporadic classic variant renal AML with tumor thrombus in the renal vein and inferior vena cava (IVC) during pregnancy is rare. We report a case of complex surgical treatment during pregnancy with normal childbirth. A 27 -year-old woman with bilateral AML was faced with large asymptomatic AML and IVC thrombus at 24 weeks of gestation. The magnetic resonance imaging showed that the tumor thrombus had fatty signal. The tumor and IVC thrombus were treated by laparoscopic nephrectomy and open tumor thrombectomy during pregnancy. The patient underwent term spontaneous vaginal delivery at 39 weeks of gestation smoothly and the neonate was in good health. Successful retroperitoneal laparoscopic nephrectomy with open tumor thrombectomy during pregnancy is possible in dealing with invasive renal AML for pregnant patients. Renal AML requires close follow-up during pregnancy. And detailed consult with urologists is necessary before pregnancy.
\end{abstract}

Keywords: Pregnancy; renal angiomyolipoma (AML); surgical treatment; tumor thrombus; case report

Submitted Dec 06, 2019. Accepted for publication Dec 27, 2019.

doi: $10.21037 /$ tau.2019.12.43

View this article at: http://dx.doi.org/10.21037/tau.2019.12.43

\section{Introduction}

Renal angiomyolipoma (AML) is a kind of benign renal mesenchymal tumor occurring mainly in adults. Renal AML can occur sporadically or associated with tuberous sclerosis complex (TSC) and pulmonary lymphangioleiomyomatosis (LAM). Most of them are classic variants with abnormally thick-walled vessels, varying composition of smooth-musclelike cells, adipocyte-like cells and epithelioid cells (sparse or less than 10\%) in pathology (1). Compared with epithelioid variant, classic renal AML is rare in local invasion extending into renal vein, inferior vena cava (IVC) or right atrium. In addition, pregnancy and estrogen therapy are discovered to promote the growth of renal AML. A larger renal AML is more likely to occur hemorrhage or spontaneous tumor rupture, while AML with tumor thrombus can be much more dangerous. The surgical treatment is recommended when renal AML is larger than $4 \mathrm{~cm}$ in diameter or with complications. The treatment in pregnant AML patients can be a real dilemma to protect both patients and their fetuses, especially in pregnant patients with IVC tumor thrombus. Only a few cases have been reported in dealing with the IVC tumor thrombus during pregnancy $(2,3)$.

We report on a case of a pregnant woman with large right renal AML and IVC tumor thrombus, which was treated by laparoscopic nephrectomy and open thrombectomy during 26 weeks of gestation. To our knowledge, this is the first case reported using laparoscope assisted way in dealing with renal AML with IVC tumor thrombus during pregnancy. In the case report, the influence of pregnancy on renal AML and the preliminary experience in managing specific pregnant patients with renal AML vena cava thrombus was discussed.

\section{Case report}

A 27-year-old nulliparous woman was referred to our 


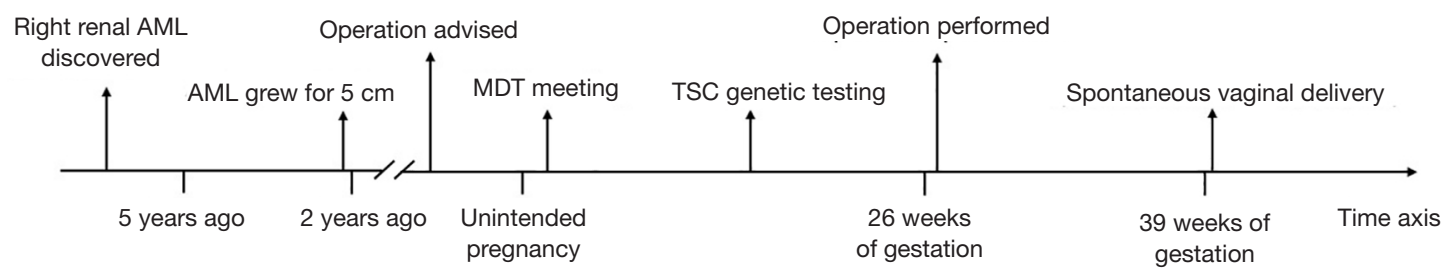

Figure 1 The timeline picture of historical and current information from this episode of care. AML, angiomyolipoma; MDT, multidisciplinary team; TSC, tuberous sclerosis complex.

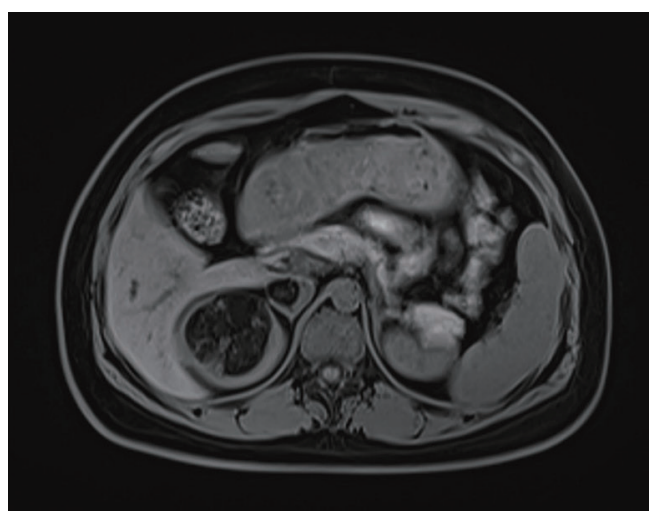

Figure 2 Renal MRI at 24 weeks of gestation: hyposignal fatsat T1W transverse view of the renal AML and IVC thrombus. The AML and thrombus had fatty signal. MRI, magnetic resonance imaging; AML, angiomyolipoma; IVC, inferior vena cava.

hospital's obstetrics department for an unintended pregnancy with a history of bilateral asymptomatic renal AML. And at 24 weeks of gestation, the largest right AML was about $7.4 \mathrm{~cm} \times 5.3 \mathrm{~cm} \times 4.7 \mathrm{~cm}$ with a definite right renal vein and IVC tumor thrombus, diagnosed using a renal magnetic resonance imaging (MRI).

The young woman with a history of right renal AML for more than 5 years was strongly suggested to be followed closely. She previously had been lost to further follow-up until discovering the grown right AML for more than $5 \mathrm{~cm}$, 2 years earlier. And then she was medically advised to do a laparoscopic operation. She refused surgical treatment and demanded conservative treatment again even when a renal MRI showed suspicious tumor involvement of right renal vein. A multidisciplinary team (MDT) meeting had been hold after the unintended pregnancy and the suggestion of terminating the pregnancy was given to her for the high risk of tumor rupture during the gestation. She refused and completed the tuberous sclerosis complex (TSC) genetic testing which came back with negative results. She was well informed and made her decision to take the operation, making every effort to protect the 26-weeks fetus (Figure 1).

The largest right renal AML measured $7.4 \mathrm{~cm}$ across its larger axis. The tumor thrombus was located in the right renal vein extending into intrahepatic segment of the IVC, level II IVC thrombus according to the Mayo classification (4), and presented a fatty signal on MRI (high T1W, high T2W, low fatsat T2W and slightly low DWI) (Figure 2).

Because of the high risk of tumor rupture during the third trimester of pregnancy and the size of the tumor thrombus, the decision was made to perform an operation of retroperitoneal laparoscopic nephrectomy with open tumor thrombectomy.

Detailed informed consent was given by the patient and dexamethasone was given intramuscularly in a regular dose (four doses of $5 \mathrm{mg}, 12$ hours apart) for accelerating fetal lung maturity. Surgical intensive care unit and neonatal intensive care unit were well prepared for the surgery in case of emergency.

Under general anesthesia, retroperitoneal laparoscopic nephrectomy was performed in a left lateral decubitus position as usual. The right renal artery and upper ureter were clipped using Hem-o-lok clips, and then cut off. The right renal vein, the retro-hepatic IVC and the back side of IVC were exposed using laparoscopic dissecting forceps and ultrasonic scalpel. Two short hepatic vein and the right adrenal vein were exposed and ligated. After resection of the right adrenal gland, intraoperative laparoscopic ultrasound was used in detecting the location of the IVC thrombus. With the ultrasound guidance, a vessel tourniquet was placed above the proximal thrombus. Another two vessel tourniquets were placed around the left renal vein and the caudal IVC, respectively. Then open tumor thrombectomy was performed in an oblique supine position for better surgical exposure. The caudal IVC, left renal vein and cephalic IVC were sequentially clamped. Next, the IVC wall was cut, removing the right kidney with IVC thrombus (Figure 3) and the right adrenal gland. After taking out the above tissues, the IVC lumen was checked and closed using 5-0 polypropylene suture. IVC clamping 


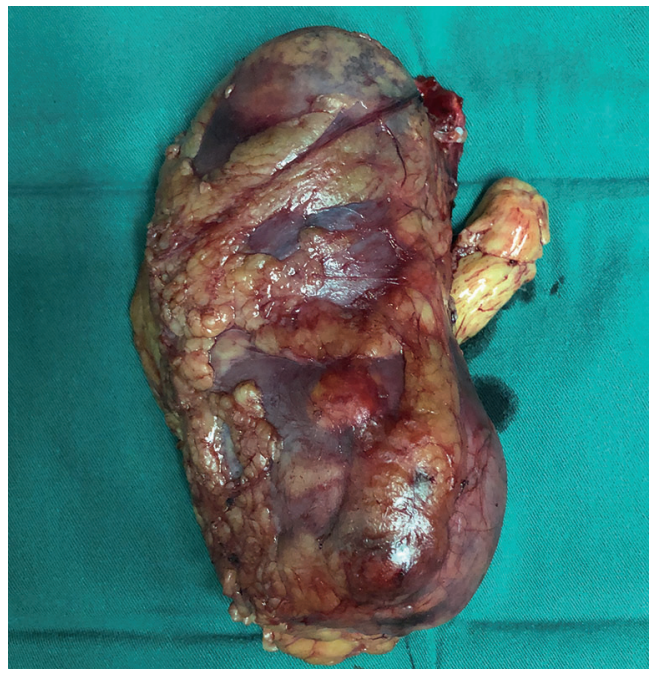

Figure 3 The renal AML and IVC thrombus. The renal mass was on the upper pole and it was $8 \mathrm{~cm}$ across its larger axis. AML, angiomyolipoma; IVC, inferior vena cava.

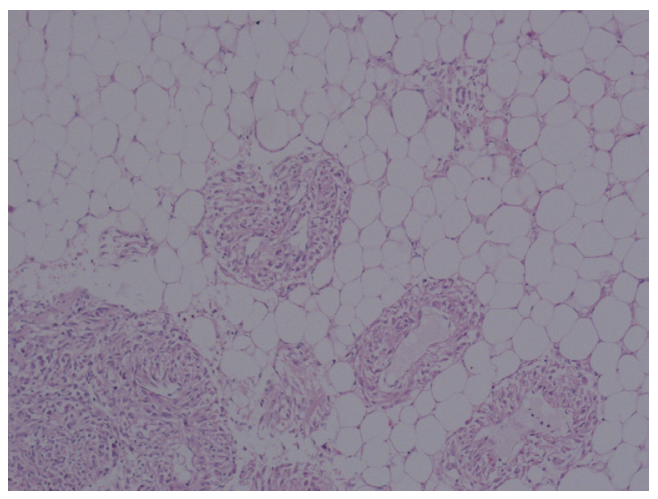

Figure 4 Pathologic findings of the renal mass: sporadic classic variant triphasic angiomyolipoma. Hematoxylin-eosin $(\times 100$, original magnification).

lasted for 8 minutes and was well tolerated. Intraoperative transesophageal echocardiography was used to confirm that the IVC and right atrium were thrombus free. During the operation, the fetal heartbeat and the patients' blood pressure were continually monitored and signified normalcy.

During the recovery period, the patient was normal in fetal monitoring without uterine contraction. Broadspectrum antibiotic and low molecular weight heparin were used for 5 days postoperatively until the temperature and postoperative activity level were continually normal. Drainage tube and catheter were removed at the fourth day postoperatively. Pathological examination revealed a sporadic classic variant renal AML (Figure 4). One week after operation, the patient went back home with everything normal according to the abdominal ultrasonographic examination. Finally, the patient underwent term spontaneous vaginal delivery at 39 weeks of gestation smoothly. And a healthy male neonate weighing 3,545 g was delivered with Apgar scores of 10 at both 1 and 5 minutes. The patient recovered well and the following-up abdominal CT scan showed normal postoperative changes in right retroperitoneum with the stable asymptomatic left renal AML. The neonate was in good health with detailed follow-up in the department of pediatrics and developed normally according to scales of infant development.

\section{Discussion}

To our knowledge, this is the third published case of surgical management to deal with renal AML with IVC tumor thrombus during pregnancy, but the first case reported using laparoscope assisted way. The first case published in 2011 reported successful AML isolated thrombectomy in managing a free-floating AML vena cava thrombus during pregnancy (2). The other case published in late 2011 reported successful open nephrectomy with the removal of the vena cava thrombus during pregnancy (3). In this case, we faced with bilateral asymptomatic renal AML, of which the largest one was more than $7 \mathrm{~cm}$ and companied with IVC thrombus. Considering the demand of the patient, we believe the retroperitoneal laparoscopic nephrectomy with open tumor thrombectomy during the second trimester of pregnancy, without medical radiation exposure and following uncertain risk, is the best solution.

The successful performance of retroperitoneal laparoscopic nephrectomy with open tumor thrombectomy during pregnancy is a practice of retroperitoneal laparoscopic technique in pregnant patient for both fetal and maternal safety, which is more difficult than published cases (5). Although the urologists are aware that selective angioembolization and nephron-sparing surgery are methods in treating renal AML during pregnancy (5), they cannot be performed and fail to deal with the sustaining risk of IVC tumor thrombus. It is confirmed that general surgery is safe for pregnant patient, and laparoscopic surgery using carbon dioxide pneumoperitoneum is safe for fetus (6).

The following points need to be considered: Using ultrasonography and MRI is enough in AML diagnosis, follow-up during pregnancy and routine preoperative 
examination, which can avoid extra medical radiation exposure; an obstetric consultation or MDT meeting about maternal physiology and anatomy is needed before the decision of non-obstetric surgery (7); obstetrician and neonatologist are needed to standby and monitor maternal and fetal condition during operation; surgical intensive care unit and cardiac surgery are prepared for emergency; under general anesthesia, hypotension and anesthesia depth need to be controlled to prevent uterine ischemia and abortion; careful manipulation of urologist is required during operation, while experienced maternal and fetal monitor of obstetrician is required postoperatively; a thorough discussion with the patient and explanation of risks for each available treatments are needed to insure well informed consent.

What's more, fetal pulmonary maturation and vena cava compression syndrome should be considered before operation. Corticosteroids can be used before operation to hasten fetal pulmonary maturation. Shorter vena cava clamping time pressure, agents used during operation and hydration before operation can improve the patient's tolerance and maintain suitable mean arterial pressure. Anticoagulants were used after operation balancing the risk of thrombosis and hemorrhage. After managing the bleeding and coagulation dysfunction during perioperative period, a spontaneous pregnancy and delivery without the use of anticoagulants is recommended.

Renal AML with IVC thrombus has been reported in a lot of cases. However, renal AML with IVC thrombus during pregnancy is infrequent. These cases are lack of standardized clinical practice guideline for treatment, while our case is intended to give an available treatment choice. There are also cases reporting renal cell carcinoma (RCC) with IVC thrombus during pregnancy (8). It is reported that tumor thrombus of RCC can extend to the right atrium, level IV IVC thrombus according to the Mayo classification. A higher level of tumor thrombus can put spontaneous delivery at unacceptable risk and put surgical treatment at imperative strategy. Therefore, the operative treatment during pregnancy is to be considered but individualized depending on patient's condition. Since the successful performance of retroperitoneal laparoscopic nephrectomy with open tumor thrombectomy during pregnancy in managing $\mathrm{AML}$ with IVC thrombus, surgical management for RCC with level I and level II IVC thrombus can be considered during pregnancy to pursue spontaneous delivery.

Our preliminary experience in managing specific pregnant patients with renal AML vena cava thrombus has limitations. The IVC tumor thrombi dealt during pregnancy are coming from right renal tumor and developed from the right renal vein in published cases. The experience dealing with left renal tumor with IVC thrombus during pregnancy is limited. Considering the uterus is usually in dextrorotation physiologically during late pregnancy, surgical position of a right lateral decubitus under general anesthesia needs to be cautious for potential uterine ischemia and requires tighter fetal and uterine monitoring during operation. Besides, an IVC thrombus developed from the left renal tumor can also be more difficult in surgical anatomy. Our preliminary experience may not be applicable in managing pregnant patients with left renal AML vena cava thrombus.

Since the renal AML is a monitorable benign tumor, the best way is to avoid the dilemma of renal AML with IVC thrombus during pregnancy. It is important that close follow-up, health education and professional suggestions are emphasized to renal AML patients before they are ready for pregnancy. If the renal AML meets surgical indication (more than $4 \mathrm{~cm}$ in size or with other complications), surgical treatment is suggested before pregnancy. The female sex hormones during pregnancy can promote the growth of renal AMLs. Estrogen receptor and progesterone receptor expressions are discovered in renal AML. This may explain the pathogenesis of rapid AML growth during pregnancy and further research is needed. The patient in this case is negative in TSC genetic testing but has bilateral renal AML. And during the follow-up and pregnancy, only the right larger AML grew rapidly. This may imply the treatment strategy for patients with multiple renal AML preparing for pregnancy and the mechanism of the likely heterogeneity in multiple renal AML need further research.

Lastly, we demonstrate that it is possible to manage renal AML with IVC tumor thrombus by retroperitoneal laparoscopic nephrectomy with open tumor thrombectomy during pregnancy for excellent maternal and fetal outcome. Renal AML needs serious assessment and treatment before and during pregnancy.

\section{Acknowledgments}

Funding: None.

\section{Footnote}

Conflicts of Interest: All authors have completed the ICMJE uniform disclosure form (available at http://dx.doi. 
org/10.21037/tau.2019.12.43). The authors have no conflicts of interest to declare.

Ethical Statement: The authors are accountable for all aspects of the work in ensuring that questions related to the accuracy or integrity of any part of the work are appropriately investigated and resolved. All procedures performed in studies involving human participants were approved by the Biomedical Research Ethics Committee of Peking University First Hospital and were in accordance with the 1964 Helsinki declaration and its later amendments or comparable ethical standards. Written informed consent was obtained from the patients for publication of this manuscript and any accompanying images.

Open Access Statement: This is an Open Access article distributed in accordance with the Creative Commons Attribution-NonCommercial-NoDerivs 4.0 International License (CC BY-NC-ND 4.0), which permits the noncommercial replication and distribution of the article with the strict proviso that no changes or edits are made and the original work is properly cited (including links to both the formal publication through the relevant DOI and the license). See: https://creativecommons.org/licenses/by-nc$\mathrm{nd} / 4.0 \%$.

\section{References}

1. Aydin H, Magi-Galluzzi C, Lane BR, et al. Renal

Cite this article as: Zhang Z, Liu Z, Yao L, Zhang Q, He $Z$. Surgical management of angiomyolipoma with vena cava thrombus during pregnancy: a case report. Transl Androl Urol 2020;9(2):807-811. doi: 10.21037/tau.2019.12.43 angiomyolipoma: clinicopathologic study of 194 cases with emphasis on the epithelioid histology and tuberous sclerosis association. Am J Surg Pathol 2009;33:289-97.

2. Lopater J, Hartung O, Bretelle F, et al. Management of angiomyolipoma vena cava thrombus during pregnancy. Obstet Gynecol 2011;117:440-3.

3. Govednik-Horny C, Atkins M. Angiomyolipoma with vascular invasion during pregnancy. Ann Vasc Surg 2011;25:1138.e1139-13.

4. Blute ML, Leibovich BC, Lohse CM, et al. The Mayo Clinic experience with surgical management, complications and outcome for patients with renal cell carcinoma and venous tumour thrombus. BJU Int 2004;94:33-41.

5. Dell'Atti L, Borghi C, Galosi AB. Laparoscopic Approach in Management of Renal Cell Carcinoma During Pregnancy: State of the Art. Clin Genitourin Cancer 2019;17:e822-30.

6. Lee SH, Lee JY, Choi YY, et al. Laparoscopic appendectomy versus open appendectomy for suspected appendicitis during pregnancy: a systematic review and updated meta-analysis. BMC Surg 2019;19:41.

7. ACOG Committee Opinion No. 775: Nonobstetric Surgery During Pregnancy. Obstet Gynecol 2019;133:e285-6.

8. Ghanney EC, Cavallo JA, Levin MA, et al. Renal cell carcinoma with inferior vena cava thrombus extending to the right atrium diagnosed during pregnancy. Ther Adv Urol 2017;9:155-9. 